\title{
WATER BODY EXTRACTION USING MIXTURE ANALYSIS TECHNIQUES AND MATHEMATICAL MORPHOLOGY
}

\author{
GERMÁN TORRIJOS. C, MIGUEL A. ÁVILA. A \& J ULIÁN R. CAMARGO L \\ Engineering Faculty, Universidad Distrital Francisco José de Caldas, Bogotá, Colombia
}

\begin{abstract}
In this work, Remote Sensing was used together with Digital Image Processing (DIP) for the extraction of the water body (Sao Paulo dam), using an orbital image. The techniques chosen or selected were mixture analysis, which have as theoretical basis the spectral proportion in the resolution element and aim to generate image-fraction corresponding to the pure components present in a resolution element, in the case of vegetation, soil and water, and Mathematical Morphology (MM), which is suitable for processing the images taking into account the geometry of the sets contained in them. The results obtained showed that the techniques used were effective, proving that they can be used to detect cartographic features in digital images and that they can be used later in conventional processes of cartographic updating.

KEYWORDS: Digital Image Processing, Mathematical Morphology
\end{abstract}

Received: Oct 06, 2020; Accepted: Oct 26, 2020; Published: Dec 01, 2020; Paper Id.: IJMPERDOCT202079

\section{INTRODUCTION}

Brazilian hydrography allows diversified economic exploration, such as drinking water sources, agricultural irrigation and energy production. In addition to the above, Brazil's hydrographic network is considered the most extensive in the world, with approximately $55,500 \mathrm{~km} 2$.

The data obtained by remote sensing prove to be an important tool for diagnostic detection and activity planning. The large volume of data provided by natural resources satellites and the development of computer science, have led to the emergence of several techniques for digital image processing. These images can be considered a source of data for studies, where the detection of alterations of characteristics of the earth's surface is one of the main tasks in the area of cartography.

The mixture analysis is a way to verify which are the primary or pure components of the pixel and as a result are originated fraction magnets, which represent the proportions of the components in the spectral mixture. Mathematical Morphology is one of the tools that allows the extraction of those objects of interest, it comprises the area that studies topological and structural properties of the objects from their images, having as objective to describe quantitatively their geometric structures. 


\section{MIXTURE ANALYSIS}

The mixture analysis is a way to verify which are the primary components or pure components of the pixel, where the pixel is the result of the mixture or linear combination of several components considered pure in its formation, where it contains information about the proportion and spectral response of each component.

The spectral mixing phenomenon occurs due to the fact that the radiance recorded by a sensor is the sum of the radiances of all materials within the instantaneous visa field (IFOV) of the sensor. The mixture analysis uses a spectral combination model to describe and separate the contribution of each material within the pixel, where it is based on the assumption that the spectral response of each pixel can be modeled as a linear combination of pure components (Novo \& Shimabukuro, 1994).

The spectral blend model was developed to decompose the multispectral image into three components, according to the "land use" of each pixel represented in the image.

According to (Ponzoni \& Shimabukuro, 2007), the linear spectral mixture model can be written as:

$r_{i}=a *$ vege $_{i}+b$ soil $_{i}+c *$ water $_{i}+e(1)$

Where, $r_{i}$ Mean spectral reflectance for the i-th spectral band, a, b, and c Spectral reflectance of the component in the pixel for the spectral band; $v_{e g} e_{i}$, soil $_{i}$ water $_{i}$, proportion value of each component in the pixel, $e$ Estimation error for the i-band. The result of the linear composition is the generation of three fraction images, each one containing information of the three constituents of the pixel: vegetation, soil and water.

\section{MATHEMATICAL MORPHOLOGY}

Mathematical Morphology began in the 1960s, more precisely between 1964 and 1968, at the Ecole Supérieure des Mines in Paris, as a result of studies carried out by a group of researchers led by George Matheron and Jean Serra.

The word Morphology originates from the Greek and means treatise (logos) of the forms (morphos ). The original purpose was to analyze geometrical structures, in microscopic images of rock samples or materials, and to relate the results to physical properties of the materials (BANON; \& BARRERA; 1994).

According to SOILE (1999), Mathematical Morphology can be defined as a theory for the analysis of spatial structures. It is called Morphology because it tends to analyze the shape of objects. It is mathematical in the sense that the analysis is based on the theory of sets, geometric integrals and Boolean algebra. Therefore, Mathematical Morphology is not only a theory, but also a powerful image analysis technique.

\section{MATERIALS AND METHODS}

In this work three CBERS 2 image clippings, CCD sensor instrument, acquired in 2007, were used. The images were obtained from the website of the National Institute for Space Research (INPE - www.inp.br). From the available bands, bands 3, 4, and 5 (red, near infrared, and panchromatic) were chosen because they present information with greater spectral contrast of the objects.

The manipulation of the images was performed through the Idrisi software, which is a Digital Image Processing (DIP) software, developed by the Graduate School of Geography at Clark University and designed for the analysis of 
geographic data in matrix format, EASTMAN (2006), and the Mathematical Morphology (MM) Toolbox, developed by SDC Information Systems, which runs in conjunction with MATLAB software.

After acquiring the image through the National Institute of Space Research, it was cut for the location of the work area, as shown in Figure 1.
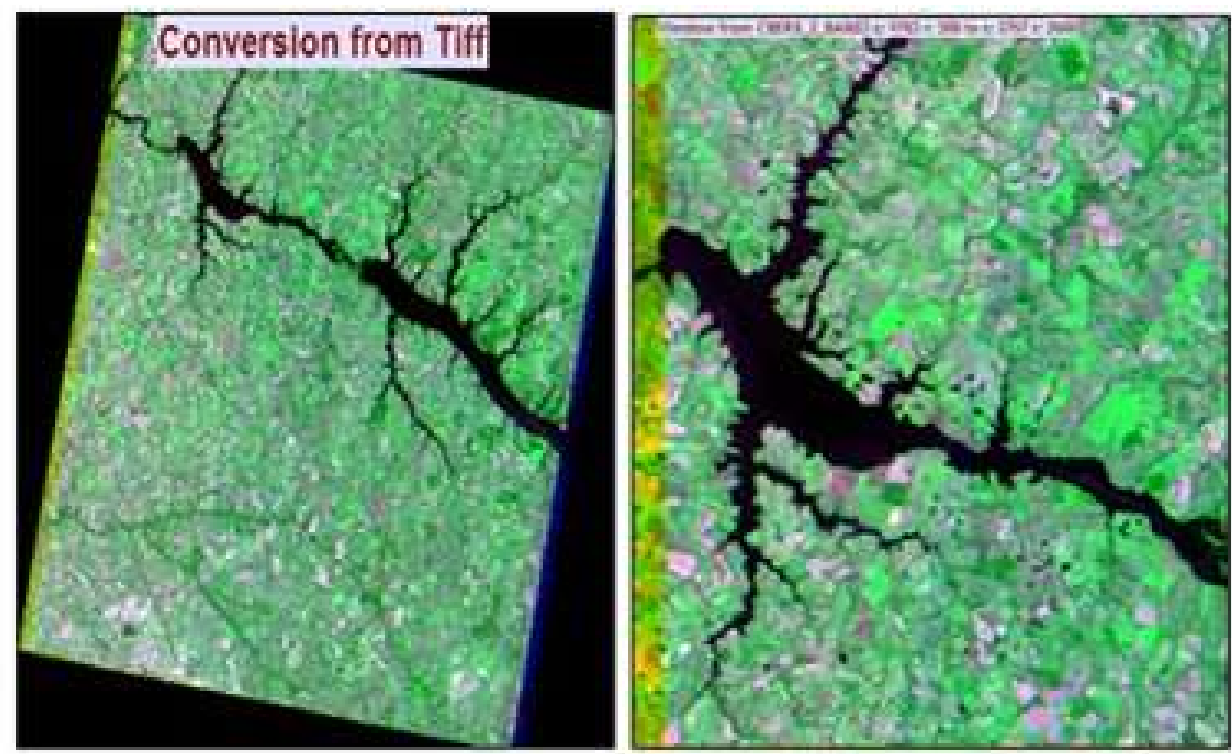

Figure 1: (a) original image obtained through INPE, (b) cropped image

Subsequently, the process of obtaining the fraction images began. First, it was necessary to create a spectral signature file, as pure as possible as a representation of the pixels of the objects to be identified. To do this, it was necessary to acquire samples of water, soil and vegetation, in places where there was no mixture or combination of them. The regions from which the samples were collected were chosen in the following way:

- Vegetation: area containing light green pixels, without containing shade variability;

- Water body: area containing black pixels;

- Soil: area containing pixels of magenta color and without tonality variability.

Due to the great variability of the analyzed image, mainly when the types of vegetation present, in different stages of the image, some difficulty was found in the identification of pixels that were considered pure for each of the samples.

To create the fraction images, we start from an image with the same parameters of the original image. Later, the selected polygons were used as samples in the image to generate a signature file. This file was defined by analyzing the histograms and the number of pixels for each class, which corresponded to at least 30 pixels per class.

This is how the fraction images resulting from the mixture analysis model originated, based on the linear mixture model, which assumes that the pixel is the result of the mixture of several components considered pure in its formation. Once the fraction images are obtained, the one that best represented the water body is chosen and the morphological processing begins. 


\section{RESULTS}

Figure 2 below shows the generated fraction images of the pure water, vegetation and soil components, respectively.

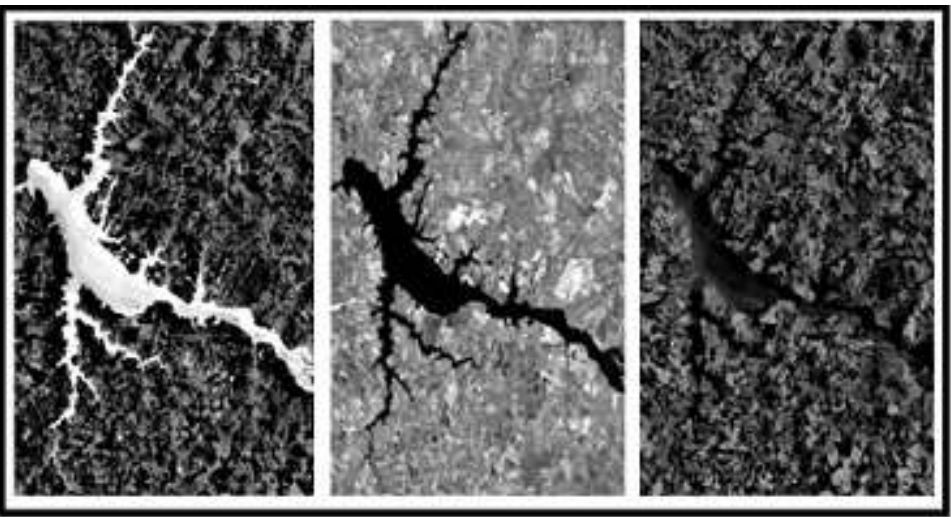

a.

b.

c.

Figure 2: Fractionated images, for pure water components (a), vegetation (b), soil (c).

By analyzing the components in shades of gray, it is possible to notice that the pixels with attributes closer to one (1) white represent the objects corresponding to the pure component in question. For example, in the pure vegetation component, the pixels with attributes closer to one (1) represent the vegetation; the less vegetation present, the darker the pixels. The fraction image that best differentiates the water body from other objects and the pure water component. For this reason this image was selected for the extraction of the water body using Mathematical Morphology. The image was converted into gray levels using the Irfan Veiw software, in order to use it with the Matlab® software.

The first step after converting the image to gray levels was to initiate morphological processing, where preprocessing was performed on the image trying to improve visual quality, increasing the contrast between the objects of interest and the other objects and decreasing the difference between the background objects. The limits used are altered according to the present interest in each image and the brightness values of the same, most of these limits were obtained through the analysis of the histogram. As a first enhancement processing was applied the addm operator, whose function is to create a new image by adding two others, with saturation. The limit chosen was 60 . The result is shown in Figure 3.

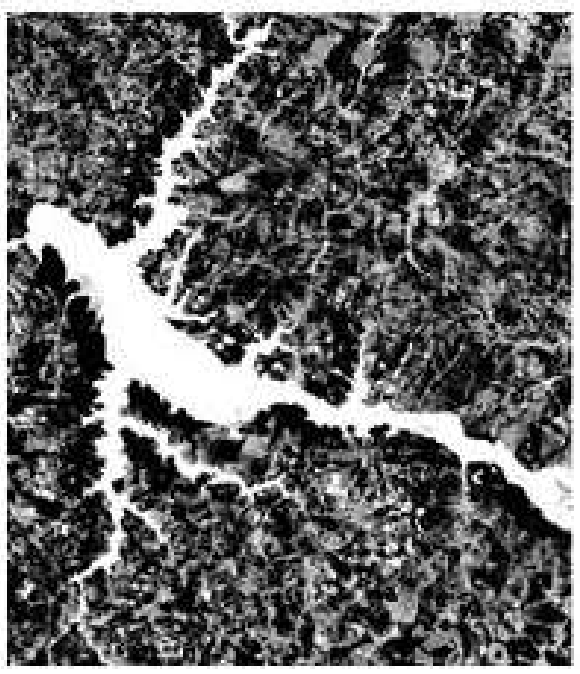

Figure 3: Application of the addm operator. 
In the sequence the operator used was the hdome, with limit 30, that operator aims to alter the brightness values of the image. The result of applying this operator is shown in Figure 4.

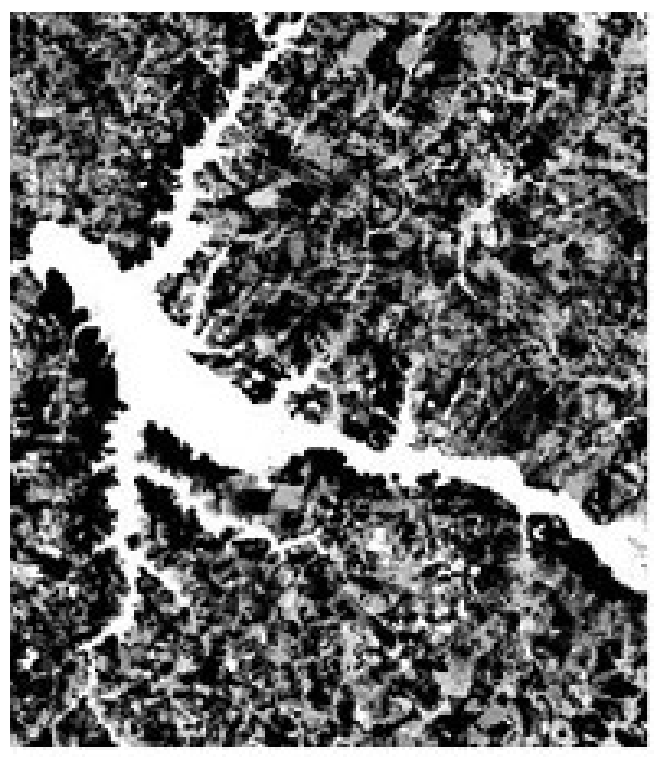

Figure 4: Result of the application of the hdome operator.

After the pre-processing stage, the image was binary through the binary operator, with a limit of 205 . This value was obtained through the analysis of the histogram of the image. This value was obtained through the analysis of the histogram of the image. The values of the pixels that were below the stipulated limit assumed a value of zero (0) corresponding to the color black and the values that were above the stipulated limit assumed a value of one (1) white. The result of applying this operator is illustrated in Figure 5.

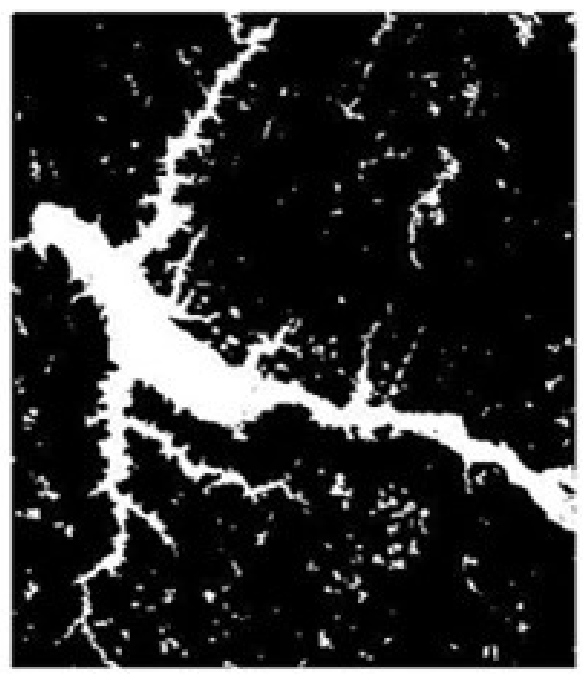

Figure 5: Result of the application of the binary operator.

From figure 5, it is possible to appreciate the noises present in the image. Before eliminating them, it was necessary to dilate the image with the objective of connecting the points or regions of the part of interest and thus later remove the noises, without altering the object of interest. The operator used for the dilation was the dil, with the secross structuring element. Figure 6 illustrates the result of the application of the operator. 


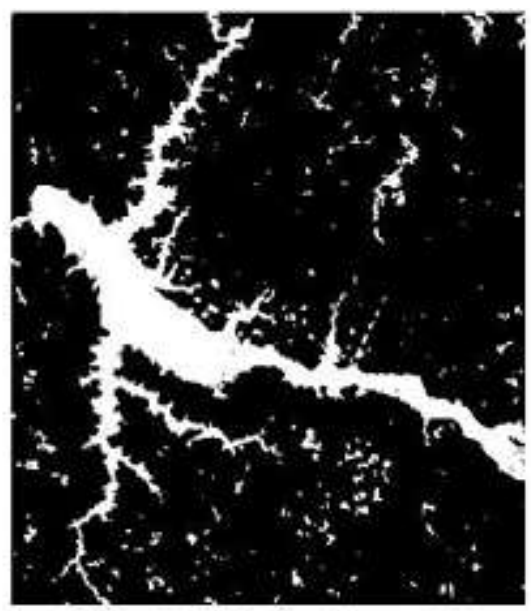

Figure 6: Result of the dil- secross operator application.

Figure 6 shows the persistence of noise, for this reason the areaopen operator was applied with a limit of 5000, with the aim of eliminating noise in the image, because this operator aims to remove in a binary image any component with area less than the limit value found. The result of the application of this operator is shown in Figure 7.

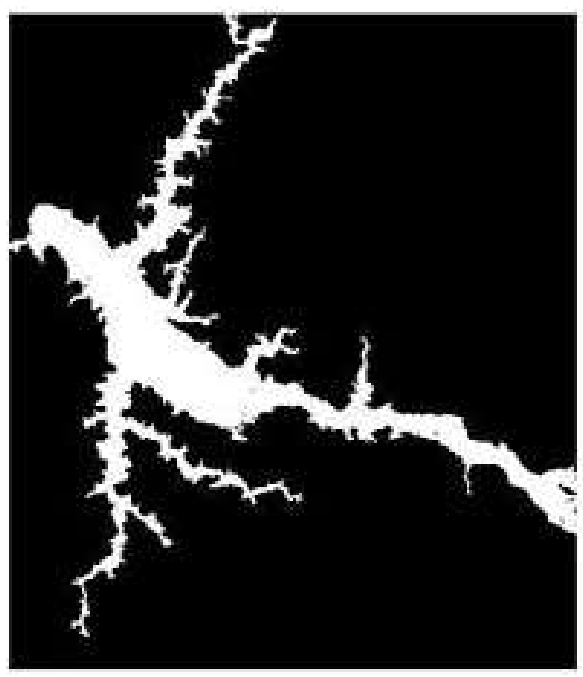

Figure 7: Result of the application of the areaopen operator.

Then the image was "eroded" by the application of the "ero" operator with the structuring element "secross", returning to its original form. In figure 8 . 


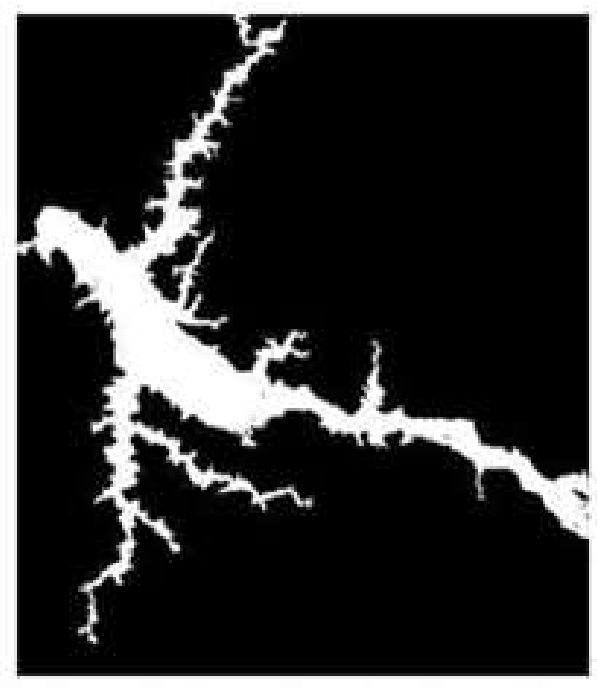

Figure 8: Result of the operator's application er(secross).

From figure 8 it can be stated that the "geometric characteristics" of the extracted object were preserved maintaining its original properties and features. Figure 9 shows the extracted water body superimposed on the original image.

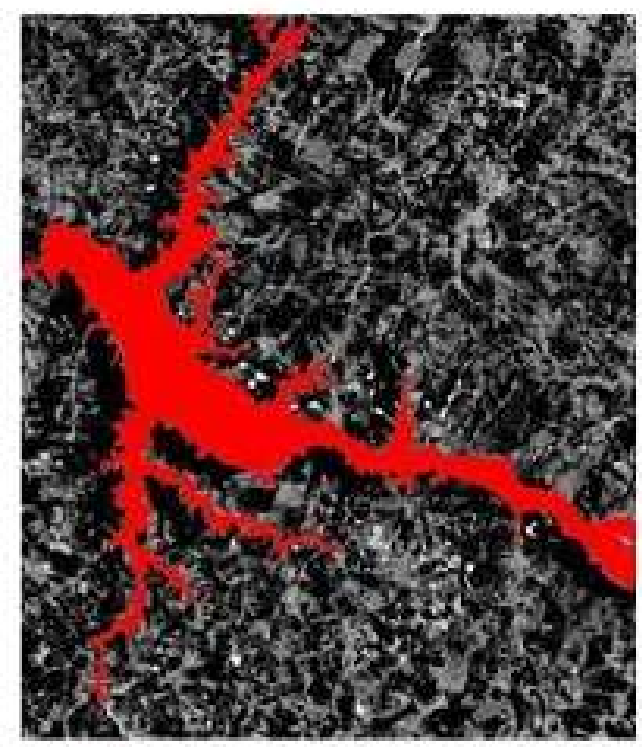

Figure 9: Result of the over-positioning.

\section{CONCLUSIONS}

Through the results obtained and presented, it is possible to conclude that the tools used for mixture analysis together with Mathematical Morphology are effective techniques regarding the extraction of cartographic characteristics.

It is highlighted that the selection of the adequate operators and limits contributed fundamentally to the detection of the characteristics of the water body, in which the result indicates that the Morphological processes used were adequate. An important fact to be highlighted is that the application of morphological operators did not alter the position of the characteristics of the water body. 
The extraction of water bodies is important in the area of cartography because these characteristics can be used in conventional processes of updating cartographic products that have, for example, the delimitation of the extensions of dams, lakes or water reservoirs for hydroelectric plants, among other applications.

\section{REFERENCES}

1. BANON, G. J. F. \& BARRERA, J. (1994) Bases da Morfologia Matemática para análise de imagens binárias - Recife, UFPE.

2. EASTMAN, J R. (2006). IDRISI Andes: Guide to GIS and image processing. Manual versión 15. Worcester: Clark University.

3. NOVO, E. M. L. M. \& SHIMABUKURO, Y. E.(1994). Spectral mixture analysis of inland tropical waters. International Journal of Remote Sensing, p. 1351-1356.

4. PONZONI, F. J. \& SHIMABUKURO, Y. E. (2007). Sensoriamento Remoto no Estudo da Vegetação. São José dos Campos: Editora Parêntese. 